\title{
GenoChemetic Strategy for Derivatization of the Violacein Natural Product Scaffold
}

\author{
Hung-En Lai, Alan M. C. Obled, Soo Mei Chee, Rhodri M. Morgan, Rosemary Lynch, Sunil V. Sharma,
} Simon J. Moore, Karen M. Polizzi, Rebecca J. M. Goss,* and Paul S. Freemont*

Cite This: https://doi.org/10.1021/acschembio.1c00483

Read Online

ACCESS |

山ll Metrics \& More

回 Article Recommendations

S1 Supporting Information

ABSTRACT: Natural products and their analogues are often challenging to synthesize due to their complex scaffolds and embedded functional groups. Solely relying on engineering the biosynthesis of natural products may lead to limited compound diversity. Integrating synthetic biology with synthetic chemistry allows rapid access to much more diverse portfolios of xenobiotic compounds, which may accelerate the discovery of new therapeutics. As a proof-of-concept, by supplementing an Escherichia coli strain expressing the violacein biosynthesis pathway with 5-bromo-tryptophan in vitro or tryptophan 7-halogenase RebH in vivo, six halogenated analogues of violacein or

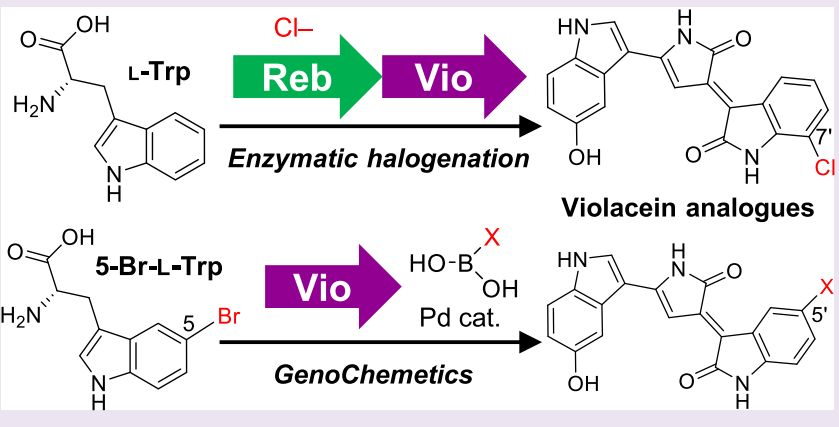
deoxyviolacein were generated, demonstrating the promiscuity of the violacein biosynthesis pathway. Furthermore, 20 new derivatives were generated from 5-brominated violacein analogues via the Suzuki-Miyaura cross-coupling reaction directly using the crude extract without prior purification. Herein we demonstrate a flexible and rapid approach to access a diverse chemical space that can be applied to a wide range of natural product scaffolds.

\section{INTRODUCTION}

The total synthesis of natural products and their analogues has often been challenging and costly due to their structural complexities; ${ }^{1}$ however, greater chemical space may be accessed through the utilization of synthetic chemistry methodologies and reagents compared with employing enzyme-catalyzed biosynthesis alone. Combining biosynthesis with chemical synthesis represents a powerful approach for rapidly generating new analogues of complex natural products and libraries of chemical derivatives suitable for screening and structureactivity relationship (SAR) assays, ${ }^{2,3}$ leading to the discovery of new and more potent compounds. The generation of such analogues can enable improvement in the bioactivity and bioavailability, as illustrated, for example, by analogues of the "last-resort" antibiotic vancomycin, with a >200-fold improvement of potency compared with vancomycin in resistant Enterococci strains. ${ }^{4}$ In another study, the addition of a sterically unhindered primary amine group to a Gram-positive antibiotic deoxynybomycin expanded its antimicrobial activity to multidrug-resistant Gram-negative pathogenic strains. ${ }^{5}$

As a proof-of-concept, we have focused on the violacein biosynthetic pathway. Violacein (1), a violet pigment first isolated from the bacterium Chromobacterium violaceum, is part of the bisindole biosynthetic family that utilizes L-tryptophan as the starting substrate. The violacein pathway is encoded within a conserved operon of five genes (vioABCDE), whose gene products catalyze a 14 -electron oxidative biosynthesis pathway. ${ }^{6}$
The bisindole biosynthetic pathways have attracted considerable interest because of their therapeutic potential for medical applications, including antimicrobial, antiviral, trypanocidal, and antitumorigenic properties. ${ }^{7}$ The violacein biosynthetic pathway also produces deoxyviolacein (2) as a byproduct, and the colored properties of violacein and deoxyviolacein make them interesting targets for natural product pathway engineering, such as promoter library screening, ${ }^{8}$ CRISPR-based multiplex transcriptional regulation, ${ }^{9}$ or diverting pathway flux via ribosomal-binding site (RBS) engineering.

A study on oxyviolacein, a hydroxylated analogue of violacein generated by feeding exogenous 5-hydroxy-L-tryptophan, ${ }^{11}$ showed bioactivity against a collection of pathogenic bacteria and fungi strains, ${ }^{12}$ suggesting that violacein analogues may be a good starting point for developing more potent antibiotics. A similar rationally designed precursor-directed biosynthetic strategy has also showed considerable success in generating analogues of flavonoids. ${ }^{13}$ Other examples of using altered biosynthetic pathways for analogue generation include the use of various enzyme homologues from bisindole pathways, ${ }^{14,15}$

Received: June 23, 2021 


\section{Enzymatic tryptophan halogenation + hybrid biosynthesis}
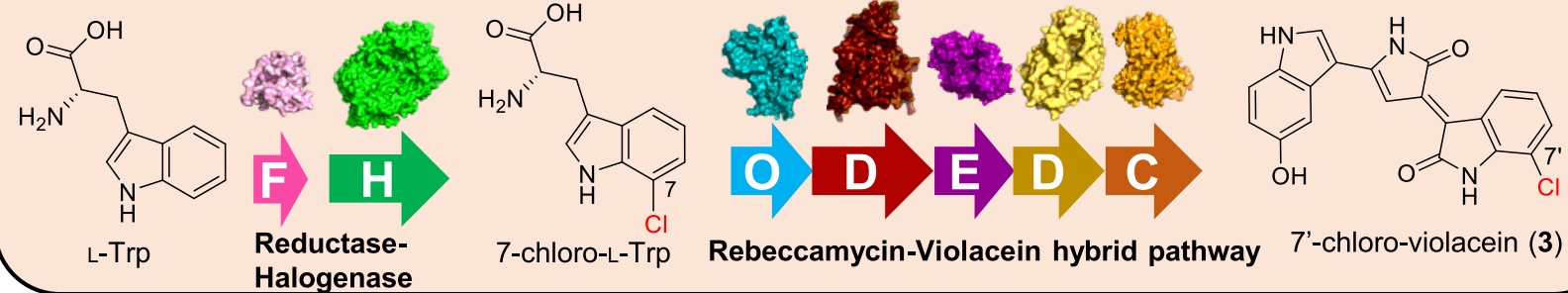

7'-chloro-violacein (3)

\section{GenoChemetics: in vivo biosynthesis + direct cross-coupling}

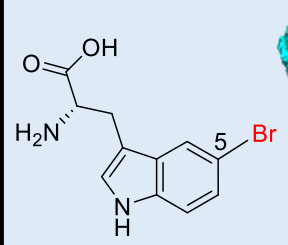

5-bromo-L-Trp

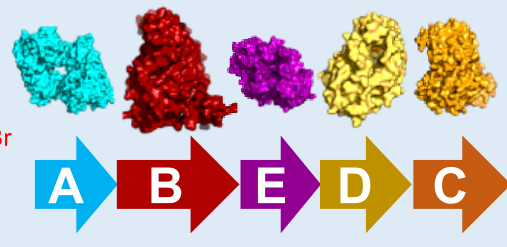

Violacein pathway

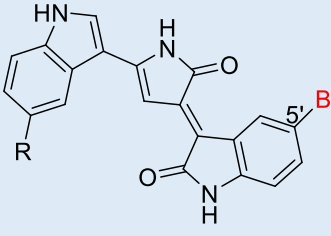

$\mathrm{R}=\mathrm{OH} \quad$ 5'-bromo-violacein (5) $\mathrm{R}=\mathrm{H} \quad$ 5'-bromo-deoxyviolacein $(6)$

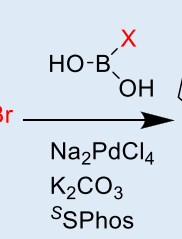

(6)

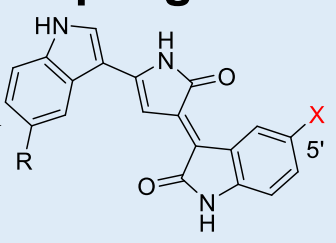

Cross-coupling derivatized products

Figure 1. Schematics of the GenoChemetic approach used in this study. Analogues of violacein (1) or deoxyviolacein (2) can be generated via the in vivo halogenation of tryptophan by expressing flavin reductase RebF and tryptophan halogenase RebH with the rest of the violacein pathway. Further derivatization via Suzuki-Miyaura cross-coupling lead to new synthetic analogues. Available enzyme structures are shown and correspond to VioA (PDB 6G2P), VioD (PDB 3C4A), VioE (PDB 3BMZ), and RebH (PDB 2E4G) as well as models generated in silico by Phyre2 (http://www.sbg.bio. ic.ac.uk/phyre2) for RebO, RebD, VioB, VioC, and RebF.

interconversion of the biosynthetically related novobiocin and clorobiocin aminocoumarin antibiotics, ${ }^{16}$ introduction of the halogenase from the hormaomycin pathway to generate clorobiocin analogues, ${ }^{17}$ and generation of chlorinated monoterpene indole alkaloids. ${ }^{18}$ A prominent strategy in generating natural product analogues is GenoChemetics, where reactive halogen handles are installed via engineered biosynthetic pathways on natural products, which are subsequently derivatized via living-culture-optimized cross-coupling reactions, resulting in analogues of the antibiotic pacidamycin. ${ }^{19}$ Although the total chemical synthesis of violacein ${ }^{20,21}$ and some substituted analogues ${ }^{22,23}$ has been reported, these syntheses are challenging and low-yielding. Pathway manipulation could represent a more flexible, sustainable, and rapid approach for generating violacein analogues. In this study, we have applied such approaches to generate 26 new violacein or deoxyviolacein analogues via a combination of pathway engineering enabling the enzymatic halogenation of the starting substrate tryptophan, feeding brominated tryptophan, and further derivatization using Suzuki-Miyaura cross-coupling directly in crude extracts (Figure 1).

\section{RESULTS AND DISCUSSION}

VioA, the first enzyme in the violacein biosynthesis pathway, oxidizes the substrate L-tryptophan to form the first pathway intermediate indole-3-pyruvic acid imine (IPA imine). To determine whether VioA would accept different tryptophan analogues as substrates, we purified VioA (Figure S1) and carried out VioA enzyme kinetic assays against various substrate analogues including L-tryptophan (TRP), 4-fluoro-D,L-tryptophan (4FT), 5-methyl-D,L-tryptophan (5MeT), 6-fluoro-D,Ltryptophan (6FT), and 7-methyl-D,L-tryptophan (7MeT). These substrates were chosen to probe the effect of substituted positions of tryptophan indole rings on VioA-substrate kinetics. $4 \mathrm{FT}, 6 \mathrm{FT}$, and $7 \mathrm{MeT}$ were shown to exhibit a wide range of analogue conversion yields in our previous study on substratefed violacein analogues, ${ }^{24}$ whereas VioA was shown to have a higher activity against $5 \mathrm{MeT}$ than TRP. ${ }^{25}$ The kinetics of VioA against L-tryptophan $\left(k_{\text {cat }}=3.04 \mathrm{~s}^{-1}, 95 \% \mathrm{CI}\right.$ [2.17 to 5.33]; $K_{\mathrm{M}}$ $=447 \mu \mathrm{M}, 95 \% \mathrm{CI}$ [255 to 977], $\left.k_{\mathrm{cat}} / K_{\mathrm{M}}=6.80 \mathrm{~s}^{-1} \mathrm{mM}^{-1}\right)$ have been previously determined with both UV-monitored substrate depletion and coupled peroxidase assays, although only the kinetic data generated from the substrate depletion assay were reported $\left(k_{\mathrm{cat}}=3.38 \pm 0.32 \mathrm{~s}^{-1}, K_{\mathrm{M}}=31 \pm 11 \mu \mathrm{M}\right){ }^{6}$ The discrepancy between the reported values and our data could be due to the difference in the assay conditions, the kinetic models chosen, or the multiple reaction steps involved in the coupled peroxidase assay. Among the substituted tryptophan substrates, 6FT exhibited the highest $k_{\text {cat }} / K_{\mathrm{M}}$ value $\left(13.9 \mathrm{~s}^{-1} \mathrm{mM}^{-1}\right)$, followed by $7 \mathrm{MeT}\left(11.2 \mathrm{~s}^{-1} \mathrm{mM}^{-1}\right), 5 \mathrm{MeT}\left(3.18 \mathrm{~s}^{-1} \mathrm{mM}^{-1}\right)$, and 4FT $\left(<0.0658 \mathrm{~s}^{-1} \mathrm{mM}^{-1}\right)$ (Figure 2a,b, Table S1). We cannot accurately estimate the $k_{\text {cat }} / K_{\mathrm{M}}$ value of VioA against 4FT because the best-fit value of $K_{\mathrm{M}}, 8.307 \mathrm{mM}$ is beyond the range of substrate concentration tested (up to $5 \mathrm{mM}$ ). Nonetheless, this shows that VioA exhibits a much higher activity against 6FT compared with other substituted tryptophan analogues tested, indicating a strong preference at the six-substituted position. Interestingly, our data show that VioA has only $47 \%$ relative activity against $5 \mathrm{MeT}$ compared with L-tryptophan, in contrast with previous characterization, ${ }^{25}$ but this is perhaps due to the difference in kinetic models chosen for our data (substrate inhibition) as opposed to the MichaelisMenten model in the previous study.

To determine if the substrate kinetics differences were due to alterations in the substrate binding, we next solved the crystal structures of apo VioA (PDB 6ESD) and VioA complexed with TRP (PDB 6G2P), 4FT (PDB 6FW7), 5MeT (PDB 6FW8), 6FT (PDB 6FW9), and 7MeT (PDB 6FWA) with resolutions ranging from 2.4 to $3.0 \AA$ (Figure $2 \mathrm{c}$,d, data statistics in Table $\mathrm{S} 2$ ). Our apo VioA structure is nearly identical to the published 
a

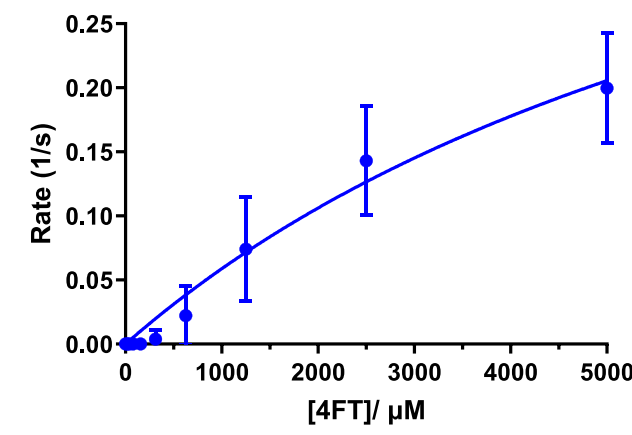

b

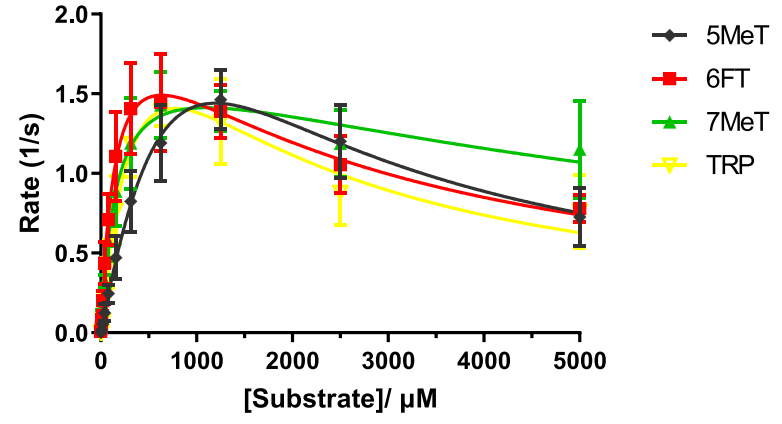

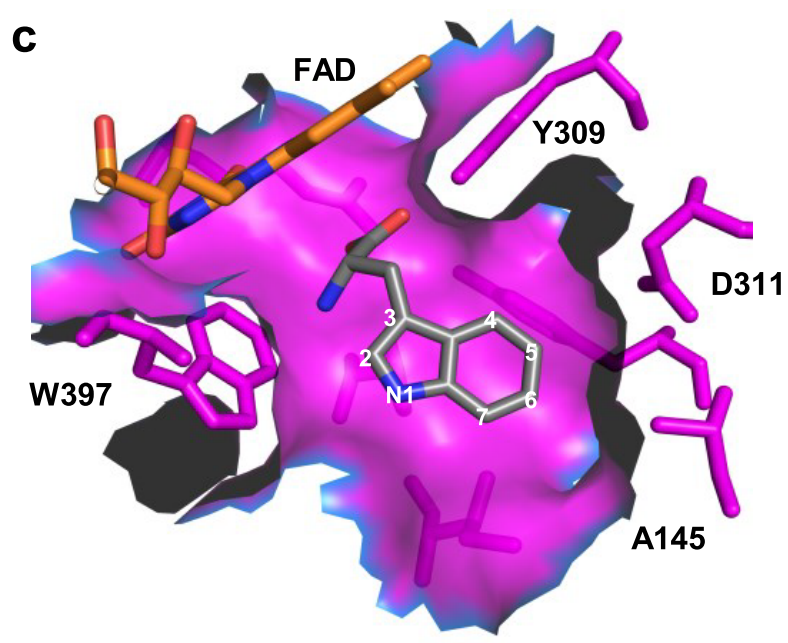

d

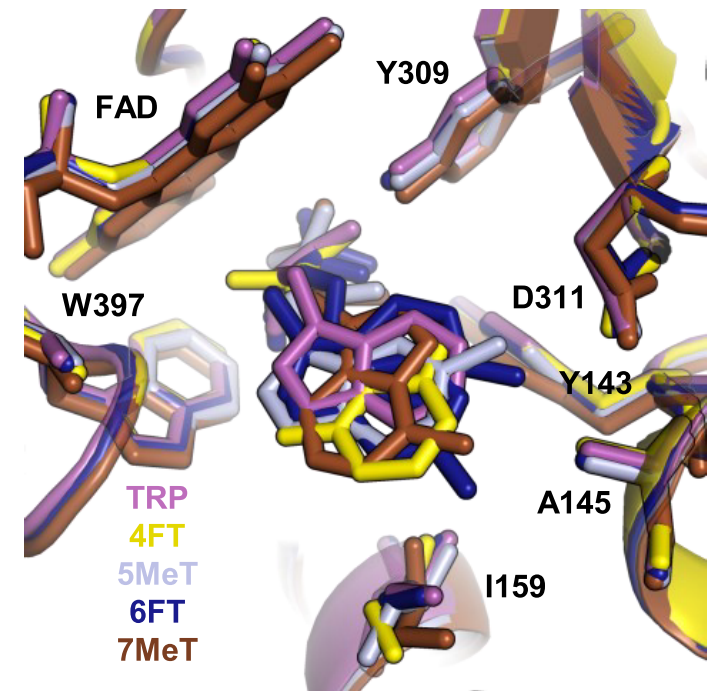

Figure 2. VioA substrate kinetics and structure. (a) Michaelis-Menten model is fitted on the VioA kinetics data against 4-fluoro-D,L-tryptophan (4FT). (b) Substrate inhibition model fitted on VioA kinetics data against L-tryptophan (TRP), 5-methyl-D,L-tryptophan (5MeT), 6-fluoro-D,Ltryptophan $(6 \mathrm{FT})$, and 7-methyl-D,L-tryptophan $(7 \mathrm{MeT})$. Data represent the mean and error bars represent the SD of three independent experiments carried out on three separate days. Kinetic parameters are in Table S1. (c) Binding site pocket showing the space enclosed by the residues (magenta) around L-tryptophan (gray with elemental color). The carbon positions of the tryptophan indole ring are labeled. (d) Superimposition of the active sites of VioA structures complexed with L-tryptophan (TRP, magenta), 4-fluoro-L-tryptophan (4FT, yellow), 5-methyl-L-tryptophan (5MeT, light gray), 6-fluoro-L-tryptophan (6FT, dark blue), and 7-methyl-L-tryptophan (7MeT, brown) in stick representation. Side chains surrounding the tryptophan ligand are labeled. Note that 6 FT has two conformers fitted in the density map. (See Figure S2.)

apo VioA structure $($ PDB 5G3T, rmsd $=0.940 \AA)$, whereas our tryptophan-bound structure (PDB 6G2P) is also very similar to both the inhibitor-bound $\operatorname{VioA}^{25}$ (PDB 5G3U, 0.586 $\AA$ ) and the tryptophan-bound C395A VioA mutant ${ }^{26}$ (PDB 5ZBD, 0.689 $\AA)$. The four-substituted tryptophan-substrate-bound VioA structures are also virtually identical to the tryptophan-bound VioA structure, with an rmsd in the range of 0.304 to $0.419 \AA$. The active site of VioA reveals several key residues surrounding the $\mathrm{C} 4$ to $\mathrm{C} 7$ positions of the tryptophan substrate indole ring. Of the four positions, $\mathrm{C} 4$ and $\mathrm{C} 7$ have the most space around the binding site pocket compared with C5 and C6 due to Asp311 and Ala145 side chains (Figure 2c), suggesting that VioA would have the lowest affinity for tryptophan analogues with substituent groups at the C5 and C6 positions. However, the interatomic distances between the respective $\mathrm{C} \alpha$ atom or amine nitrogen atom and the $\mathrm{N} 5$ of the $\mathrm{FAD}$ cofactor are conserved for all of the analogues (between 3.93 and $4.69 \AA$ for the $\mathrm{C} \alpha$ atom and 3.62 and $5.63 \AA$ for amine nitrogen) and are similar to that of the TRP-bound structure ( $3.93 \AA$ for $\mathrm{C} \alpha$ atom and $5.07 \AA$ for amine nitrogen) (Figure S2). This showed that all of the analogues can bind VioA in a catalytically active but slightly different conformation. Interestingly, the hydrogen bonding between Arg64 and Tyr309 and the carboxylate group of all substrate analogues is conserved, as are the positions of the other active-site residues (Figure 2d). Our data clearly show that the VioA active site can flexibly accommodate a variety of tryptophan substrate analogues for catalysis.

Recently, we have generated a wide range of violacein and deoxyviolacein analogues from $E$. coli cells that were expressing a synthetic violacein biosynthesis pathway vio $A B C D E$ with added substituted tryptophans, and these crude extracts were tested against the malarial parasite Plasmodium falciparum. ${ }^{24}$ Because of the promiscuity of the enzymes involved in the biosynthesis of violacein, we rationalized that a combination of enzymes from closely related bisindole biosynthesis pathways could generate compatible tryptophan analogues, leading to other interesting new-to-nature analogues of violacein that might otherwise be difficult to synthesize. Rebeccamycin is a closely related bisindole where the biosynthetic pathway contains several genes, including rebO (Q8KHS0) and $r e b D$ (Q8KHV6), which are homologous to vioA (Q9S3V1) and vioB (Q9S3V0), respectively. In addition, the pathway also consists of a tryptophan 7-halogenase $\mathrm{RebH}$ and its associated flavin reductase RebF that are responsible for generating 7-chloro-L- 
a

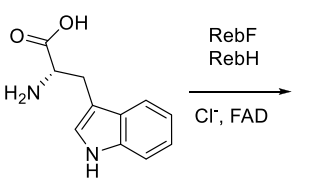

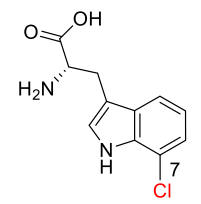

L-Tryptophan
7-Chloro-L-Tryptophan

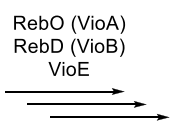

FAD, Heme b

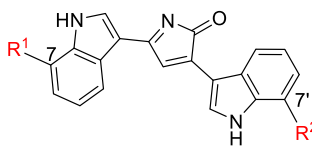

$\mathrm{R} 1=\mathrm{H}, \mathrm{R} 2=\mathrm{Cl} \quad 7^{\prime}$-Chloro-PDV $\mathrm{R} 1=\mathrm{Cl}, \mathrm{R} 2=\mathrm{H}$ 7-Chloro-PDV $\mathrm{R} 1=\mathrm{R} 2=\mathrm{Cl} \quad 7,7^{\prime}$-Dichloro-PDV
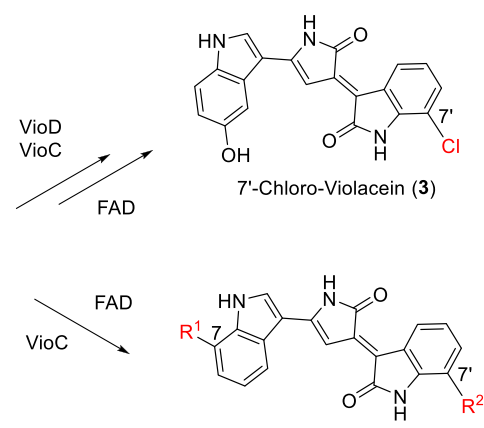

$\mathrm{R} 1=\mathrm{H}, \mathrm{R} 2=\mathrm{Cl} \quad 7^{\prime}-$ Chloro-DV (4a) $\mathrm{R} 1=\mathrm{Cl}, \mathrm{R} 2=\mathrm{H} \quad$ 7-Chloro-DV (4b) $\mathrm{R} 1=\mathrm{R} 2=\mathrm{Cl} \quad 7,7^{\prime}$-Dichloro-DV (4c) b

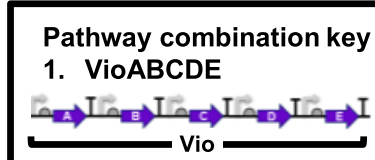

\section{RebOD+VioCDE}

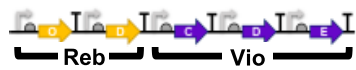

\section{RebFH+VioABCDE}

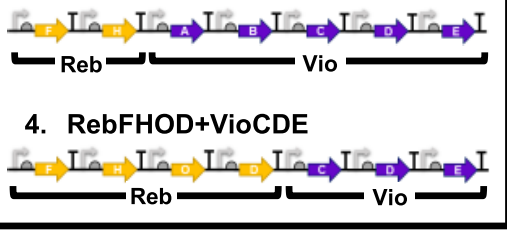

C ${ }_{\times 10^{6}}^{\text {EIC }}$ Pathway 1: VioABCDE

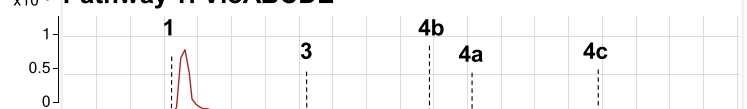

$10^{6}$ Pathway 2: RebOD+VioCDE

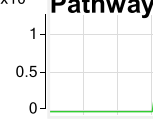

$\times 10^{6}$ Pathway 3: RebFH+VioABCDE

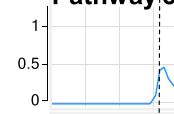

$\times 10^{6}$ Pathway 4: RebFHOD+VioCDE

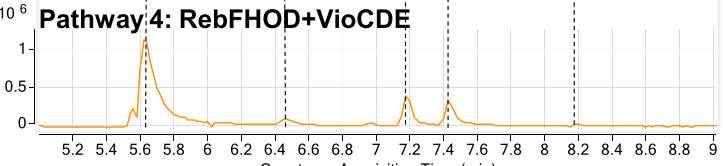

d

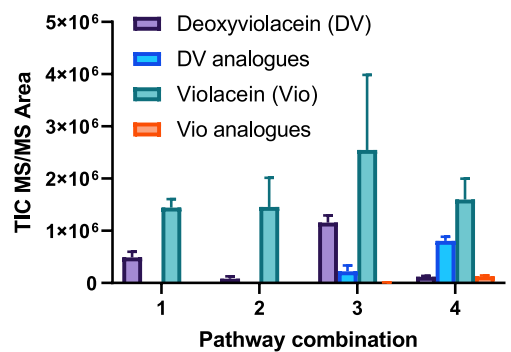

e

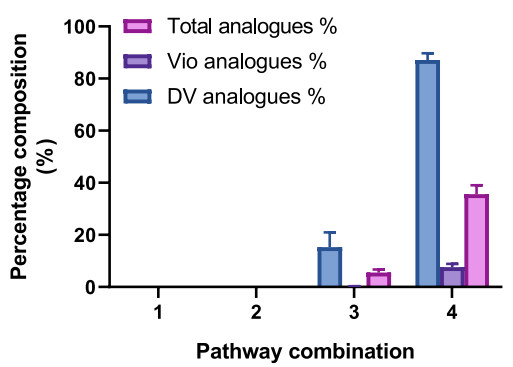

f

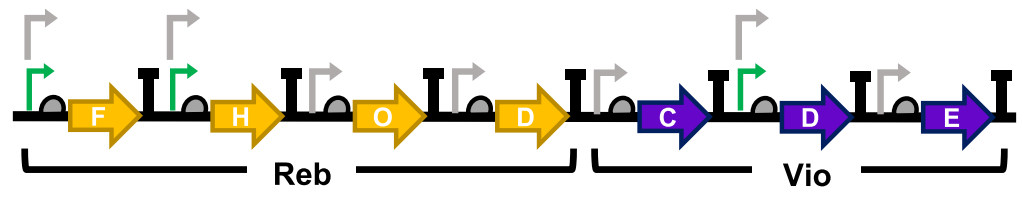

Promoter key

J23114 (weak)

J23108 (medium)

Strain-promoter combinations

\begin{tabular}{|c|c|c|c|}
\hline Strain & RebF & RebH & VioD \\
\hline S1 & J23114 & J23114 & J23114 \\
\hline S2 & J23108 & J23108 & J23108 \\
\hline S3 & J23108 & J23108 & J23114 \\
\hline
\end{tabular}

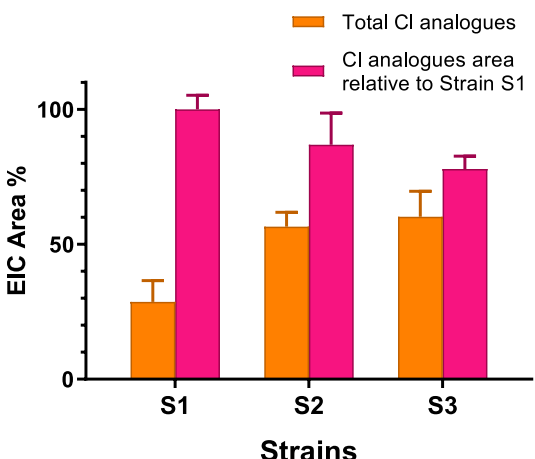

Figure 3. Generation of 7-chloro analogues of violacein and deoxyviolacein via in vivo halogenation. (a) Schematic of the hybrid rebeccamycinviolacein pathway enzymes that lead to 7 -chloro analogues of violacein (3) and deoxyviolacein (4a-c), as analyzed by LC-HRMS/MS (Table S3). (b) Four constructs constituting various combinations of violacein and rebeccamycin biosynthetic genes to generate chlorinated violacein analogues. Pathway symbols were drawn using the SBOL visual standard for biological parts. (c) Representative extracted ion chromatograms (EICs) targeting $[\mathrm{M}+\mathrm{H}]^{+} \mathrm{m} / z$ of $\mathbf{1}, \mathbf{3}$, and $\mathbf{4 a}-\mathbf{c}$ detected from ethanol extracts of $E$. coli cells harboring one of the pathway combinations in panel b. The compounds detected are shown with a dotted line to indicate the approximate retention times. Compound $\mathbf{2}$ is not shown here because it elutes at the same retention time as 3. The VioABCDE sample was run on a separate day, so the retention time of $\mathbf{1}$ is slightly later than that of the other three samples. (d) Area under curves of $\mathrm{m} / \mathrm{z}$ species extracted at the MS/MS level from the TIC of ethanol extracts from cells harboring the respective pathway combination in panel b. Vio analogue refers to 3, whereas DV analogues refer to $4 a-c$. (e) Percentage compositions of Vio and DV analogues from different E. coli strains expressing hybrid Reb/Vio pathway variants. Data show the mean and SD of biological duplicates. (f) Percentage compositions of 7-chloro analogues of violacein and deoxyviolacein extracted from E. coli expressing RebFHOD+VioCDE with different combinations of medium (J23108, green) and weak (J23114, gray) promoters controlling the expression of RebF, RebH, and VioD. Data show the mean and SD of biological triplicates. 
a<smiles>N[C@@H](Cc1c[nH]c2ccc(Br)cc12)C(=O)O</smiles>

5-bromo-L-tryptophan<smiles>[R]c1ccc2[nH]cc(C3=C/C(=C4\C(=O)Nc5ccc(Br)cc54)C(=O)N3)c2c1</smiles>

$\mathrm{R}=\mathrm{OH} \quad$ 5'-bromo-violacein (5) $\mathrm{R}=\mathrm{H} \quad 5$ '-bromo-deoxyviolacein (6)

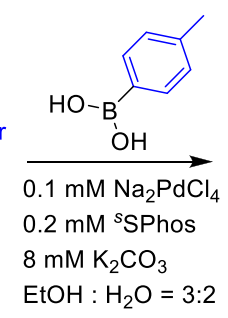

EtOH : $\mathrm{H}_{2} \mathrm{O}=3: 2$

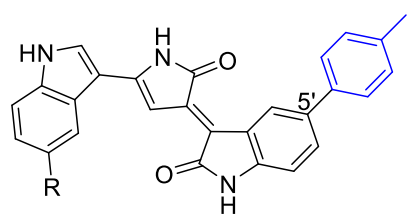

$\mathrm{R}=\mathrm{OH} \quad 5^{\prime}$-(p-tolyl)-violacein (7) $\mathrm{R}=\mathrm{H} \quad 5^{5}$-(p-tolyl)-deoxyviolacein (8)<smiles>CCOC(=O)CCC(=O)Cl</smiles><smiles>c1ccc2[nH]ccc2c1</smiles>
Indole

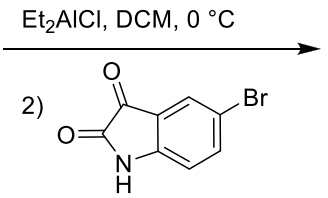

$\mathrm{NH}_{4} \mathrm{OAc}, 3 \AA \mathrm{MS}, \mathrm{PEG}-200$ $\mathrm{MW}, 200^{\circ} \mathrm{C}, 1 \mathrm{~h}$

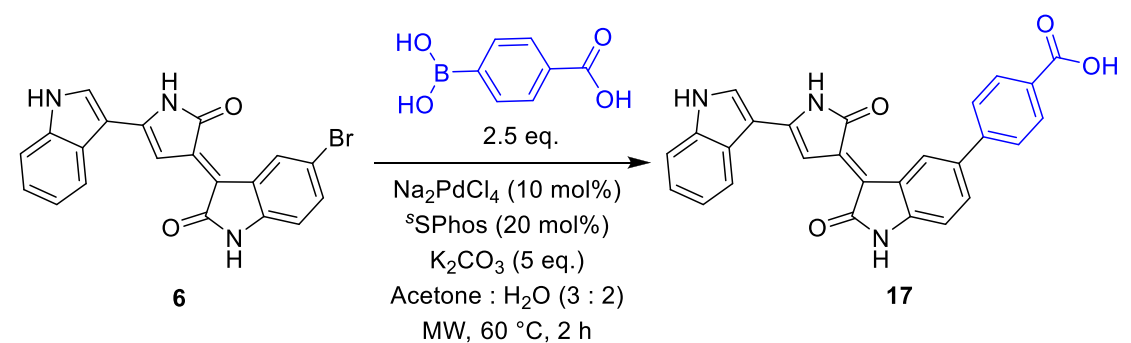

Figure 4. Further derivatization of violacein and deoxyviolacein analogues via Suzuki-Miyaura cross-coupling reactions directly in crude extracts. (a) Optimized conditions for the cross-coupling reaction directly in crude extracts. The coupling partner indicated is $p$-tolyl-boronic acid. (b) Starting from indole, $5^{\prime}$-bromo-deoxyviolacein (6) was synthesized in two steps and used for preparative scale cross-coupling to afford the product (17), which was isolated and characterized by ${ }^{1} \mathrm{H}$ NMR analysis.

tryptophan from L-tryptophan via a chloraminelysine intermediate. ${ }^{27,28}$ As a proof-of-principle, we combined the rebeccamycin (Reb) and violacein (Vio) biosynthetic operons via Golden Gate cloning 29,30 to generate C7-chlorinated violacein analogues, which we were able to successfully produce in E. coli cells (Figure 3a,b) identified via LC-HRMS/MS (Figure 3c, Table S3). We found that although the RebOD + VioCDE strain (lacking RebFH) did not produce any chlorinated analogues of violacein, it was able to functionally substitute VioAB to produce violacein and deoxyviolacein. ${ }^{6}$ Next, by comparing RebFH+VioABCDE and RebFHOD + VioCDE (where RebOD replaced VioAB) strains, we observed that RebOD increased the proportion of chlorinated analogues from 6 to 33\% when compared with VioAB (Figure 3d,e). This was likely due to the fact that RebO has a $k_{\text {cat }} / K_{\mathrm{m}}$ value that is 57 times higher for 7-Cl-L-tryptophan than for L-tryptophan, ${ }^{31}$ and thus RebO and RebD preferentially accept the 7-chloro analogue of L-tryptophan and IPA imine, respectively, producing the 7-chlorinated intermediate for downstream enzymes in the violacein biosynthesis pathway. By replacing the weak promoter J23114 for RebF and RebH with a medium strength promoter J23108 (strains S2 and S3), we observed a total analogue increase of nearly $30 \%$ (Figure 3f). We also observed an increase in $7^{\prime}$-chloro-violacein (3) by about 2.8 -fold in strain S2 with a $\mathrm{J} 23108$ promoter upstream of vioD, the biosynthetic enzyme that diverts the pathway flux toward violacein, ${ }^{6}$ whereas in strain S3, where the vioD promoter remained unchanged, the conversion to 3 increased by about two-fold compared with that in the original strain S1 (Figure S3). This shows that it is possible to fine-tune the proportion of chlorinated analogue by changing the promoter strength of pathway enzymes, and the optimization of the fermentation protocols such as the length of incubation, temperature, and media conditions might further increase both the proportion and the total amount of chlorinated analogues. ${ }^{8}$

Further derivatization, through chemical cross-coupling, of new-to-nature, halogenated natural product analogues gener- ated by a synthetic biological strain, was first demonstrated with pacidamycins. ${ }^{19}$ We applied a similar strategy by first feeding 5bromo-D,L-tryptophan to E. coli cells expressing VioABCDE to generate 5'-bromo-violacein and 5'-bromo-deoxyviolacein, which were detected by LC-HRMS/MS (Table S3). We could not find the mass corresponding to dibromo-deoxyviolacein $\left(\mathrm{C}_{20} \mathrm{H}_{11} \mathrm{~N}_{3} \mathrm{O}_{2} \mathrm{Br}_{2}\right.$, calc. $\left.[\mathrm{M}+\mathrm{H}]^{+} m / z=483.9291\right)$, 5-bromo isomers of deoxyviolacein $\left(\mathrm{C}_{20} \mathrm{H}_{12} \mathrm{~N}_{3} \mathrm{O}_{2} \mathrm{Br}\right.$, calc. $[\mathrm{M}+\mathrm{H}]^{+} \mathrm{m} / z$ $=406.0186)$, or prodeoxyviolacein $\left(\mathrm{C}_{20} \mathrm{H}_{12} \mathrm{~N}_{3} \mathrm{OBr}\right.$, calc. $[\mathrm{M}+$ $\left.\mathrm{H}]^{+} m / z=390.0237\right)$. We hypothesize that this is due to the preference of VioE in catalyzing the formation of $5^{\prime}-\mathrm{Br}$ prodeoxyviolacein, in accordance with the isomer preference previously observed with various other violacein and deoxyviolacein analogues. ${ }^{24}$ We then proceeded to screen a series of conditions that might facilitate Suzuki-Miyaura cross-coupling; however, because of low titers of 5'-bromo-violacein and 5'bromo-deoxyviolacein available in the crude extract and challenges relating to the separation of these compounds that showed a propensity for pi-stacking, the isolation of products for further characterization and bioassays of the cross-coupling product proved difficult. We thus focused on systemically identifying conditions that would allow direct cross-coupling in crude extracts, which identified the optimal conditions for derivatization. The corresponding cross-coupling products $5^{\prime}$ ( $p$-tolyl)-violacein (7) and $5^{\prime}$-( $p$-tolyl)-deoxyviolacein (8) were detected in the cross-coupling mixture by LC-HRMS/MS (Figure 4a, Table S3). Having identified the optimized conditions for cross-coupling at low concentrations, we performed the cross-coupling reaction with a selection of 15 aryl boronic acids chosen to sample a wide range of steric and electronic variations, which gave a total of 20 new cross-coupled analogues of violacein and deoxyviolacein (Table S4). We observed that whereas boronic acids with electron-donating groups (e.g., $p$-methoxyphenylboronic acid) gave better yields and MS/MS data were obtained, for those with electronwithdrawing groups (e.g., $p$-formylphenylboronic acid), only MS data were obtained due to the lower conversion and the more 
difficult electrospray ionization of the corresponding violacein analogues (Table S3). Pleasingly, even boronic acid pinacol esters were successfully converted into the corresponding violacein product (e.g., $p$-cyanophenylboronic acid pinacol ester). In summary, the optimized conditions showed that a variety of cross-coupling analogues could be synthesized without the need for purification prior to cross-coupling, allowing for rapid access to potential cross-coupling products.

The attempts to isolate brominated violaceins from the cell culture extracts proved challenging due to low titers of the desired compounds and coelution as mixtures during chromatographic purifications. Hence we decided to synthesize 5 ' -bromodeoxyviolacein (6), which will be useful as a synthetic standard and for the preparative scale cross-coupling reaction. To this end, we adapted a two-step protocol reported for the synthesis of violacein analogues. ${ }^{23}$ The condensation of the 3-acylated intermediate (characterized with ${ }^{1} \mathrm{H}$ and ${ }^{13} \mathrm{C}$ NMR in Figure S4) with 5-bromo-isatin proved challenging due to the formation of a mixture of products. However, the desired product 6 (characterized with ${ }^{1} \mathrm{H}$ NMR in Figure S5) was obtained in $15 \%$ yield after two difficult chromatographic purification steps, further highlighting the benefits of the engineered biosynthesis. The LC retention time and LC-HRMS/MS analysis of the synthetic product matched with biosynthetic $\mathbf{6}$ and confirmed the identity, as depicted (Figure S6). Furthermore, the crosscoupling of synthetic 6 with $p$-carboxyphenylboronic acid proceeded with a $>70 \%$ yield based on the LC-HRMS analysis; the product 17 was isolated ( $41 \%$ yield) from the crude reaction by an extractive workup (Figure 4b). The cross-coupling partner, $p$-carboxyphenylboronic acid, was selected for this reaction based on its polarity to improve the purification of the target compound generated. In the case of the tolyl derivative, product $\mathbf{8}$ had a markedly different retention time from starting material 6, eluting later, whereas product 17 eluted earlier than the starting material on our purification system. The product was characterized by LC-HRMS/MS (Figure S7) and ${ }^{1} \mathrm{H}$ and COSY NMR (Figure S8) analyses. These cross-coupling conditions provide a route to generate a diverse library of violacein analogues.

Combining the strengths of biosynthesis and organic synthesis, our GenoChemetic approach in generating diverse compounds has proven to be fruitful in exploring the chemical space of natural products and their derivatives. In this study, inspection of the biosynthetic assembly of violacein and crystal structure analyses of the first enzyme L-tryptophan oxidase VioA suggested that various tryptophan substrate analogues could be enzymatically processed. We utilized this finding and designed experiments combining biosynthesis and chemical synthesis to enable rapid access to six new halogenated violacein and deoxyviolacein analogues. Furthermore, we have expanded this collection of compounds further via Suzuki-Miyaura crosscoupling reactions exploiting a brominated analogue of violacein, accessing an additional series of 20 violacein or deoxyviolacein coupling products. However, the ability to carry out these synthetic diversifications selectively on the halometabolites as components of a crude cell extract is limited by the poor titers of the desired halogenated compounds and complex mixtures that render isolation more difficult than traditional synthetic routes. Therefore, further optimization of the biosynthesis of halometabolites to increase the purity or yield of the desired compounds or fine-tuning of cross-coupling conditions should pave the way toward an improved derivatization efficiency via cross-coupling in crude extracts.
Nonetheless, the combination of synthetic chemistry with synthetic biology provides a more sustainable approach with the potential for applications toward generating analogues of natural products to access greater chemical diversity rapidly and predictably.

\section{ASSOCIATED CONTENT}

\section{SI Supporting Information}

The Supporting Information is available free of charge at https://pubs.acs.org/doi/10.1021/acschembio.1c00483.

Supplemental Table S3. Violacein and deoxyviolacein analogue MSMS fragment analysis (XLSX)

Methods of all experiments. Supplemental Figures S1S8. VioA structures, LC-MS, and NMR analyses of selected compounds. Supplemental Table S1. VioAsubstrate kinetics data. Supplemental Table S2. Crystallographic data summary statistics. Supplemental Table S4. Cross-coupling products of brominated analogues of violacein and deoxyviolacein. Supplemental Protocol detailing the assembly of the rebeccamycin-violacein hybrid pathway using an EcoFlex cloning kit. Supplemental appendix containing the plasmid and protein sequence of rebeccamycin-violacein pathway plasmids. (PDF)

\section{AUTHOR INFORMATION}

\section{Corresponding Authors}

Rebecca J. M. Goss - School of Chemistry and Biomedical Sciences Research Complex, University of St Andrews, St Andrews, Fife KY16 9ST, U.K.; 이이이.org/0000-00019869-8205; Email: rjmg@st-andrews.ac.uk

Paul S. Freemont - Section of Structural and Synthetic Biology, Department of Infectious Disease, Imperial College London, London SW7 2AZ, U.K.; London Biofoundry, Imperial College Translation \& Innovation Hub, London W12 OBZ, U.K.; UK DRI Care Research and Technology Centre, Imperial College London, London W12 0NN, U.K.; (1) orcid.org/0000-00025658-8486; Email: p.freemont@imperial.ac.uk

\section{Authors}

Hung-En Lai - Section of Structural and Synthetic Biology, Department of Infectious Disease, Imperial College London, London SW7 2AZ, U.K.; Present Address: School of Biological Sciences, Victoria University of Wellington, Wellington 6012, New Zealand.; ำ orcid.org/0000-00033148-5525

Alan M. C. Obled - School of Chemistry and Biomedical Sciences Research Complex, University of St Andrews, St Andrews, Fife KY16 9ST, U.K.

Soo Mei Chee - Section of Structural and Synthetic Biology, Department of Infectious Disease, Imperial College London, London SW7 2AZ, U.K.; London Biofoundry, Imperial College Translation \& Innovation Hub, London W12 OBZ, U.K.

Rhodri M. Morgan - Department of Life Sciences, Imperial College London, London SW7 2AZ, U.K.

Rosemary Lynch - School of Chemistry and Biomedical Sciences Research Complex, University of St Andrews, St Andrews, Fife KY16 9ST, U.K.

Sunil V. Sharma - School of Chemistry and Biomedical Sciences Research Complex, University of St Andrews, St Andrews, Fife KY16 9ST, U.K. 
Simon J. Moore - Section of Structural and Synthetic Biology, Department of Infectious Disease, Imperial College London, London SW7 2AZ, U.K.; Present Address: School of Biosciences, University of Kent, Canterbury, CT7 2NJ; (1) orcid.org/0000-0002-1968-206X

Karen M. Polizzi - Department of Chemical Engineering, Imperial College London, London SW7 2AZ, U.K.; (1) orcid.org/0000-0001-5435-2667

Complete contact information is available at: https://pubs.acs.org/10.1021/acschembio.1c00483

\section{Author Contributions}

H.-E.L., S.J.M., K.M.P., S.V.S., A.M.C.O., R.J.M.G., and P.S.F. designed the study, analyzed the data, and wrote the manuscript. H.-E.L. performed experiments under the guidance of S.J.M., K.M.P., and P.S.F. A.M.C.O. performed cross-coupling reactions under the guidance of S.V.S. and R.J.M.G. R.L. prepared and characterized the synthetic $5^{\prime}$-bromo-deoxyviolacein. A.M.C.O. and S.M.C. performed the LC-HRMS/MS analysis. R.M.M. assisted with the VioA crystallography, data collection, and structure refinement. All authors read and approved the final draft of the manuscript.

\section{Notes}

The authors declare no competing financial interest.

Raw data for NMR and LC-HRMS/MS analyses are available upon request.

\section{ACKNOWLEDGMENTS}

H.E.L. was supported by an Imperial College President's Ph.D. Scholarship. We thank UKRI EPSRC (EP/K038648/1, EP/ L011573/1 to P.S.F.) and the European Union's Seventh Framework Programme (FP7/2007-2013/ERC grant agreement no. 614779 GenoChemetics to R.J.M.G.) for funding. A.M.C.O. receives funding from EPSRC CRITICAT, EP/ L016419/1. We thank all staff involved in the Diamond Light Source Beamline I04-1 for assistance with the VioA crystal data collection.

\section{REFERENCES}

(1) Kirschning, A.; Hahn, F. Merging chemical synthesis and biosynthesis: a new chapter in the total synthesis of natural products and natural product libraries. Angew. Chem., Int. Ed. 2012, 51, 40124022.

(2) Goss, R. J. M.; Shankar, S.; Fayad, A. A. The generation of "unnatural" products: synthetic biology meets synthetic chemistry. Nat. Prod. Rep. 2012, 29, 870-889.

(3) Eichner, S.; Knobloch, T.; Floss, H. G.; Fohrer, J.; Harmrolfs, K.; Hermane, J.; Schulz, A.; Sasse, F.; Spiteller, P.; Taft, F.; Kirschning, A. The interplay between mutasynthesis and semisynthesis: generation and evaluation of an ansamitocin library. Angew. Chem., Int. Ed. 2012, $51,752-757$.

(4) Okano, A.; Isley, N. A.; Boger, D. L. Peripheral modifications of $[\Psi[\mathrm{CH} 2 \mathrm{NH}] \mathrm{Tpg} 4]$ vancomycin with added synergistic mechanisms of action provide durable and potent antibiotics. Proc. Natl. Acad. Sci. U.S. A. 2017, 114, E5052-E5061.

(5) Richter, M. F.; Drown, B. S.; Riley, A. P.; Garcia, A.; Shirai, T.; Svec, R. L.; Hergenrother, P. J. Predictive compound accumulation rules yield a broad-spectrum antibiotic. Nature 2017, 545, 299-304.

(6) Balibar, C. J.; Walsh, C. T. In vitro biosynthesis of violacein from Ltryptophan by the enzymes VioA-E from Chromobacterium violaceum. Biochemistry 2006, 45, 15444-15457.

(7) Durán, M.; Ponezi, A. N.; Faljoni-Alario, A.; Teixeira, M. F. S.; Justo, G. Z.; Durán, N. Potential applications of violacein: a microbial pigment. Med. Chem. Res. 2012, 21, 1524-1532.
(8) Jones, J. A.; Vernacchio, V. R.; Lachance, D. M.; Lebovich, M.; Fu, L.; Shirke, A. N.; Schultz, V. L.; Cress, B.; Linhardt, R. J.; Koffas, M. A. G. ePathOptimize: A Combinatorial Approach for Transcriptional Balancing of Metabolic Pathways. Sci. Rep. 2015, 5, 11301.

(9) Zalatan, J. G.; Lee, M. E.; Almeida, R.; Gilbert, L. A.; Whitehead, E. H.; La Russa, M.; Tsai, J. C.; Weissman, J. S.; Dueber, J. E.; Qi, L. S.; Lim, W. A. Engineering complex synthetic transcriptional programs with CRISPR RNA scaffolds. Cell 2015, 160, 339-350.

(10) Jeschek, M.; Gerngross, D.; Panke, S. Rationally reduced libraries for combinatorial pathway optimization minimizing experimental effort. Nat. Commun. 2016, 7, 11163.

(11) Hoshino, T.; Ogasawara, N. Biosynthesis of Violacein: Evidence for the Intermediacy of 5-Hydroxy- 1 -tryptophan and the Structure of a New Pigment, Oxyviolacein, Produced by the Metabolism of 5Hydroxytryptophan. Agric. Biol. Chem. 1990, 54, 2339-2346.

(12) Wang, H.; Wang, F.; Zhu, X.; Yan, Y.; Yu, X.; Jiang, P.; Xing, X.$\mathrm{H}$. Biosynthesis and characterization of violacein, deoxyviolacein and oxyviolacein in heterologous host, and their antimicrobial activities. Biochem. Eng. J. 2012, 67, 148-155.

(13) Kufs, J. E.; Hoefgen, S.; Rautschek, J.; Bissell, A. U.; Graf, C.; Fiedler, J.; Braga, D.; Regestein, L.; Rosenbaum, M. A.; Thiele, J.; Valiante, V. Rational design of flavonoid production routes using combinatorial and precursor-directed biosynthesis. ACS Synth. Biol. 2020, 9, 1823-1832.

(14) Sánchez, C.; Zhu, L.; Braña, A. F.; Salas, A. P.; Rohr, J.; Méndez, C.; Salas, J. A. Combinatorial biosynthesis of antitumor indolocarbazole compounds. Proc. Natl. Acad. Sci. U. S. A. 2005, 102, 461-466.

(15) Du, Y.-L.; Ryan, K. S. Expansion of bisindole biosynthetic pathways by combinatorial construction. ACS Synth. Biol. 2015, 4, 682-688.

(16) Eustáquio, A. S.; Gust, B.; Li, S.-M.; Pelzer, S.; Wohlleben, W.; Chater, K. F.; Heide, L. Production of 8'-halogenated and 8'unsubstituted novobiocin derivatives in genetically engineered streptomyces coelicolor strains. Chem. Biol. 2004, 11, 1561-1572.

(17) Heide, L.; Westrich, L.; Anderle, C.; Gust, B.; Kammerer, B.; Piel, $\mathrm{J}$. Use of a halogenase of hormaomycin biosynthesis for formation of new clorobiocin analogues with 5-chloropyrrole moieties. ChemBioChem 2008, 9, 1992-1999.

(18) Runguphan, W.; Qu, X.; O'Connor, S. E. Integrating carbonhalogen bond formation into medicinal plant metabolism. Nature 2010, 468, 461-464.

(19) Roy, A. D.; Gruschow, S.; Cairns, N.; Goss, R. J. M. Gene expression enabling synthetic diversification of natural products: chemogenetic generation of pacidamycin analogs. J. Am. Chem. Soc. 2010, 132, 12243-12245.

(20) Petersen, M. T.; Nielsen, T. E. Tandem ring-closing metathesis/ isomerization reactions for the total synthesis of violacein. Org. Lett. 2013, 15, 1986-1989.

(21) Wille, G.; Steglich, W. A Short Synthesis of the Bacterial Pigments Violacein and Deoxyviolacein. Synthesis 2001, 2001, 07590762

(22) Haun, M.; Pereira, M. F.; Hoffmann, M. E.; Joyas, A.; Campos, V.; Filardi, L. D.; de Castro, S. L.; Duran, N. Bacterial chemistry. VI. Biological activities and cytotoxicity of 1,3-dihydro-2H-indol-2-one derivatives. Biol. Res. 1992, 25, 21-25.

(23) McLaughlin, E. C.; Norman, M. W.; Ko Ko, T.; Stolt, I. Threecomponent synthesis of disubstituted $2 \mathrm{H}$-pyrrol-2-ones: preparation of the violacein scaffold. Tetrahedron Lett. 2014, 55, 2609-2611.

(24) Wilkinson, M. D.; Lai, H.-E.; Freemont, P. S.; Baum, J. A Biosynthetic Platform for Antimalarial Drug Discovery. Antimicrob. Agents Chemother. 2020, 64, e02129-19.

(25) Füller, J. J.; Röpke, R.; Krausze, J.; Rennhack, K. E.; Daniel, N. P.; Blankenfeldt, W.; Schulz, S.; Jahn, D.; Moser, J. Biosynthesis of Violacein, Structure and Function of l-Tryptophan Oxidase VioA from Chromobacterium violaceum. J. Biol. Chem. 2016, 291, 20068-20084.

(26) Yamaguchi, H.; Tatsumi, M.; Takahashi, K.; Tagami, U.; Sugiki, M.; Kashiwagi, T.; Kameya, M.; Okazaki, S.; Mizukoshi, T.; Asano, Y. Protein engineering for improving the thermostability of tryptophan 
oxidase and insights from structural analysis. J. Biochem. 2018, 164, 359-367.

(27) Yeh, E.; Garneau, S.; Walsh, C. T. Robust in vitro activity of RebF and $\mathrm{RebH}$, a two-component reductase/halogenase, generating 7chlorotryptophan during rebeccamycin biosynthesis. Proc. Natl. Acad. Sci. U. S. A. 2005, 102, 3960-3965.

(28) Yeh, E.; Blasiak, L. C.; Koglin, A.; Drennan, C. L.; Walsh, C. T. Chlorination by a long-lived intermediate in the mechanism of flavindependent halogenases. Biochemistry 2007, 46, 1284-1292.

(29) Moore, S. J.; Lai, H.-E.; Kelwick, R. J. R.; Chee, S. M.; Bell, D. J.; Polizzi, K. M.; Freemont, P. S. EcoFlex: A Multifunctional MoClo Kit for E. coli Synthetic Biology. ACS Synth. Biol. 2016, 5, 1059-1069.

(30) Lai, H.-E.; Moore, S.; Polizzi, K.; Freemont, P. EcoFlex: A Multifunctional MoClo Kit for E. coli Synthetic Biology. Methods Mol. Biol. 2018, 1772, 429-444.

(31) Nishizawa, T.; Grüschow, S.; Jayamaha, D.-H. E.; NishizawaHarada, C.; Sherman, D. H. Enzymatic assembly of the bis-indole core of rebeccamycin. J. Am. Chem. Soc. 2006, 128, 724-725. 